\section{Definición mundial de consenso para el síndrome metabólico ${ }^{1}$}

El incremento del número de casos de síndrome metabólico es una de las causas de la expansión de la epidemia mundial de diabetes tipo 2 y de enfermedades cardiovasculares, según datos recientes de la Federación Internacional de Diabetes (FID). Las personas con el síndrome metabólico (20-25\% de la población mundial [1]) tienen una probabilidad tres veces mayor de sufrir un ataque cardíaco o un accidente cerebrovascular y dos veces mayor de morir por estas causas que las personas que no lo padecen (2). Por otra parte, de los casi 200 millones de personas que sufren de diabetes en el mundo, $80 \%$ mueren de enfermedades cardiovasculares (3), por lo que identificar a las personas con síndrome metabólico es un imperativo moral, médico y económico que no se debe soslayar. El diagnóstico a tiempo de esta afección permitiría aplicar intervenciones tempranas para propiciar cambios a estilos de vida más saludables, así como tratamientos preventivos que impidan las complicaciones de la diabetes y de la enfermedad cardiovascular.

Debido al enorme impacto del síndrome metabólico sobre la salud pública, en los últimos años se han realizado importantes investigaciones que han llevado a avances en el conocimiento de este complejo síndrome. Sin embargo, las múltiples definiciones existentes provocan confusión e impiden comparar entre sí muchos de los estudios realizados. Esta situación llevó a la FID a elaborar una nueva definición que pudiera tener alcance universal.

En el presente trabajo se expone la nueva definición universal de consenso presentada por la FID durante el Primer Congreso Internacional de Prediabetes y Síndrome Metabólico, celebrado en Berlín, Alemania, en abril de 2005.

\section{DEFINICIÓN APLICABLE EN TODO EL MUNDO}

La nueva definición toma en cuenta tanto las particularidades clínicas como los más recientes resultados científicos y establece criterios diagnósticos aplicables en diferentes contextos. Además, ofrece parámetros adicionales para los estudios epidemiológicos y de investigación.

Según la FID, una persona padece el síndrome metabólico si además de obesidad centroabdominal presenta uno de los siguientes signos:

- elevación de triglicéridos plasmáticos $(\geq 150$ $\mathrm{mg} / \mathrm{dL}$ ó 1,7 mmol/L) u observación de algún tratamiento específico para su control
Basado en: International Diabetes Federation. The IDF consensus worldwide definition of the metabolic syndrome. Hallado en http:/ / www.idf.org/webdata/docs/IDF_Metasyndrome_definition.pdf. Acceso el 16 de septiembre de 2005.

Palabras clave: síndrome $\mathrm{X}$ metabólico, consenso. 
- bajas concentraciones plasmáticas de colesterol ligado a lipoproteínas de alta densidad (HDL) $(<40$ $\mathrm{mg} / \mathrm{dL}$ ó $1,03 \mathrm{mmol} / \mathrm{L}$ en hombres y $<50 \mathrm{mg} / \mathrm{dL}$ ó 1,29 $\mathrm{mmol} / \mathrm{L}$ en mujeres) u observación de algún tratamiento específico para su control

- hipertensión arterial (presión sistólica $\geq 130 \mathrm{~mm}$ de $\mathrm{Hg}$ ó diastólica $\geq 85 \mathrm{~mm}$ de $\mathrm{Hg}$ ), u observación de un tratamiento con antihipertensivos

- diagnóstico previo de diabetes tipo 2 o hiperglucemia en ayunas (glucosa $\geq 100 \mathrm{mg} / \mathrm{dL}$ ó $5,6 \mathrm{mmol} / \mathrm{L}$ ). En este caso se recomienda realizar la prueba oral de tolerancia a la glucosa, aunque no es imprescindible para diagnosticar este síndrome.

La obesidad centroabdominal se determina midiendo la circunferencia de la cintura y su diagnóstico depende del sexo y del grupo étnico a los que pertenece la persona (no al país de residencia). Para ello se pueden utilizar valores de corte aceptados por consenso a partir de los resultados obtenidos en diferentes estudios, aunque se espera que investigaciones futuras permitan encontrar valores asociados más estrechamente con el riesgo de sufrir del síndrome metabólico. Los valores de referencia actualmente aceptados se presentan en el cuadro 1.

Se recomienda emplear los valores aquí expuestos para los estudios epidemiológicos y diag-

CUADRO 1. Valores de referencia para el diagnóstico de la obesidad centroabdominal

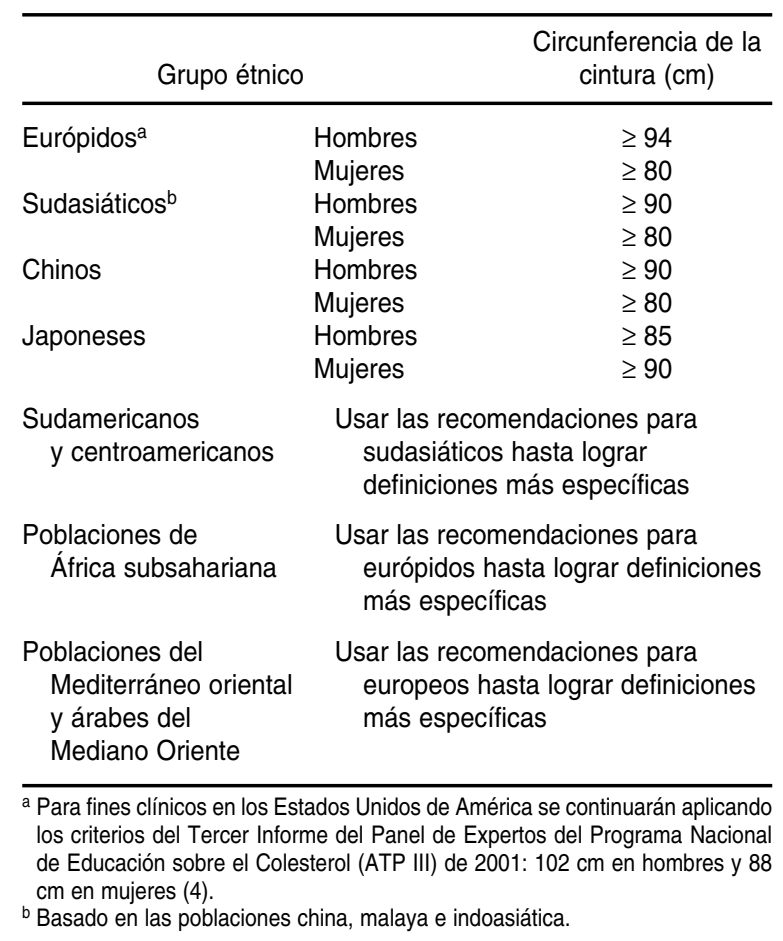

nósticos en los diferentes grupos étnicos, independientemente del país en que se encuentren. De esta forma, los criterios recomendados para los japoneses serían empleados igualmente en Japón o en las comunidades japonesas que viven en el extranjero (5). Los valores de corte empleados en la actualidad para el diagnóstico de la obesidad centroabdominal en personas de diferentes grupos étnicos en los Estados Unidos de América son más elevados que los propuestos por la FID; sin embargo, estudios epidemiológicos futuros en poblaciones de origen európido deben ayudar a definir la prevalencia del síndrome metabólico en europeos y estadounidenses según sus respectivos puntos de corte.

\section{Criterios adicionales para la investigación}

El grupo de consenso de la FID estableció parámetros adicionales (cuadro 2) que deben emplearse en estudios investigativos para determinar su capacidad de pronosticar la enfermedad cardiovascular o la diabetes. Estas investigaciones también permitirán ajustar aun más la definición de síndrome metabólico y validar la nueva definición clínica en diferentes grupos étnicos.

\section{RECOMENDACIONES PARA EL TRATAMIENTO}

Una vez establecido el diagnóstico de síndrome metabólico, el tratamiento debe ser intensivo y observarse rigurosamente para reducir el riesgo de enfermedad cardiovascular y de diabetes tipo 2 . Por lo tanto, se debe evaluar el riesgo cardiovascular de todos los pacientes (incluso su condición de fumador) y se debe establecer una intervención inicial, que puede consistir en:

- restricción moderada del consumo de calorías hasta reducir el peso corporal en 5-10\% durante el primer año

- incremento moderado de la actividad física

- cambio en la composición de la alimentación.

Cuando los cambios en el estilo de vida no son suficientes o el paciente presenta un elevado riesgo de enfermedad cardiovascular, se debe aplicar una intervención con medicamentos. Aunque se mencionen tratamientos para modular los mecanismos subyacentes del síndrome metabólico en su conjunto - que permitirían reducir el impacto de todos los factores de riesgo y las consecuencias metabólicas y cardiovasculares a largo plazo-, en la actualidad se desconocen los mecanismos de estos procesos y por lo tanto no se dispone de agentes 
CUADRO 2. Criterios metabólicos adicionales para uso en la investigación

\begin{tabular}{|c|c|}
\hline Criterio & Indicadores empleados \\
\hline $\begin{array}{l}\text { Alteraciones en la distribución de la } \\
\text { grasa corporal }\end{array}$ & $\begin{array}{l}\text { Distribución general de la grasa corporal, por absorciometría dual de rayos X } \\
\text { Distribución central de la grasa, mediante tomografía digital o resonancia magnética nuclear } \\
\text { Biomarcadores del tejido adiposo: leptina, adiponectina } \\
\text { Contenido de grasa en el hígado, mediante espectroscopia por resonancia magnética nuclear }\end{array}$ \\
\hline $\begin{array}{l}\text { Dislipidemia aterógena (además de } \\
\text { triglicéridos elevados y bajo coles- } \\
\text { terol ligado a HDL en el plasma) }\end{array}$ & $\begin{array}{l}\text { Apolipoproteína B (ApoB) o colesterol que no esté ligado a de HDL } \\
\text { Pequeñas partículas de LDL }\end{array}$ \\
\hline Disglucemia & Prueba oral de tolerancia a la glucosa \\
\hline $\begin{array}{l}\text { Resistencia a la insulina (además de } \\
\text { la glucemia elevada en ayunas) }\end{array}$ & $\begin{array}{l}\text { Concentraciones de insulina y proinsulina en ayunas } \\
\text { Índice de resistencia a la insulina, mediante el modelo matemático HOMA } \\
\text { Resistencia a la insulina, según el modelo mínimo de Bergman } \\
\text { Aumento de ácidos grasos libres (en ayunas y durante la prueba oral de tolerancia a la glucosa) } \\
\text { Sensibilidad a la glucosa (valor M) con pinzamiento }\end{array}$ \\
\hline $\begin{array}{l}\text { Desregulación vascular (además de } \\
\text { hipertensión arterial) }\end{array}$ & $\begin{array}{l}\text { Medición de la disfunción endotelial } \\
\text { Microalbuminuria }\end{array}$ \\
\hline Estado proinflamatorio & $\begin{array}{l}\text { Aumento de proteína } C \text { reactiva de alta sensibilidad (proteína amiloide A en el suero) } \\
\text { Aumento de citocinas inflamatorias (TNF- } \alpha, \mathrm{IL}-6) \\
\text { Reducción de las concentraciones de adiponectina plasmática }\end{array}$ \\
\hline Estado protrombótico & $\begin{array}{l}\text { Factores fibrinolíticos } \\
\text { Factores de la coagulación (fibrinógeno, etc.) }\end{array}$ \\
\hline Factores hormonales & Eje hipotálamo-hipófiso-suprarrenal \\
\hline
\end{tabular}

farmacológicos específicos. En su lugar se propone establecer tratamientos dirigidos a los diferentes componentes del síndrome, a manera de reducir el riesgo asociado con cada uno de ellos, lo que permitirá reducir su contribución al riesgo de enfermedad cardiovascular y diabetes. Por consiguiente, el grupo de consenso de la FID recomienda seguir pautas específicas de tratamiento para cada uno de los componentes del síndrome metabólico.

\section{Dislipidemia aterógena}

Reducir las concentraciones plasmáticas de triglicéridos, apolipoproteína B (ApoB) y colesterol que no esté ligado a HDL; elevar las de colesterol ligado a HDL; y reducir las de colesterol ligado a lipoproteínas de baja densidad (LDL).

\section{Hipertensión arterial}

La hipertensión arterial $(\geq 140 / \geq 90 \mathrm{~mm}$ de $\mathrm{Hg}$ ) se debe tratar según las recomendaciones del
Séptimo Informe del Comité Nacional Conjunto de los Estados Unidos de América para la Prevención, Detección, Evaluación y Tratamiento de la Hipertensión Arterial (6). En los pacientes diabéticos el tratamiento contra la hipertensión debe comenzar cuando la presión arterial sea $\geq 130 / 80$ $\mathrm{mm} \mathrm{Hg}$.

\section{Resistencia a la insulina e hiperglucemia}

Se espera que los medicamentos que reducen la resistencia a la insulina permitan retrasar la aparición de la diabetes tipo 2 y reducir el riesgo de enfermedad cardiovascular en pacientes con el síndrome metabólico. Entre los medicamentos estudiados se encuentran la metformina, la tiazolidinediona, la acarbosa y el orlistat (7-11).

\section{PERSPECTIVAS DE DESARROLLO}

El grupo de consenso de la FID espera que esta nueva definición - que subraya la importancia 
de la obesidad centroabdominal según el grupo étnico al que pertenece la persona- pueda ser adoptada en todo el mundo, tanto para su aplicación clínica como para estudios epidemiológicos. Esta medida podría acelerar el diagnóstico clínico de los casos de síndrome metabólico y la identificación de personas en mayor riesgo de sufrir enfermedad cardiovascular o diabetes tipo 2. La adopción de una definición mundial posibilitará comparar los datos obtenidos en diferentes estudios y ajustar esta definición a medida que se obtenga más información.

\section{SYNOPSIS}

\section{A worldwide consensus definition for the metabolic syndrome}

In recent years there have been important advances in our understanding of the metabolic syndrome. However, the multiple definitions that are used for the syndrome lead to confusion and make it hard to compare studies. Recently, the International Diabetes Federation (IDF) proposed a definition that could be applied worldwide. According to the new IDF definition, a person suffers from the metabolic syndrome if he or she has central obesity and has at least two of the following four signs: (1) elevated serum triglycerides $(\geq 150 \mathrm{mg} / \mathrm{dL}$ or $1.7 \mathrm{mmol} / \mathrm{L})$, or specific treatment for its control; (2) reduced high-density lipoprotein cholesterol $(<40 \mathrm{mg} / \mathrm{dL}$ or $1.03 \mathrm{mmol} / \mathrm{L}$ in men and < $50 \mathrm{mg} / \mathrm{dL}$ or $1.29 \mathrm{mmol} / \mathrm{L}$ in women), or specific treatment for its control; (3) arterial hypertension (systolic blood pressure $\geq 130 \mathrm{~mm} \mathrm{Hg}$ or diastolic blood pressure $\geq 85 \mathrm{~mm} \mathrm{Hg}$ ), or treatment with antihypertensives; or (4) previous diagnosis of type 2 diabetes, or elevated fasting plasma glucose $(\geq 100 \mathrm{mg} / \mathrm{dL}$ or $5.6 \mathrm{mmol} / \mathrm{L})$. If the elevated fasting plasma glucose is $\geq 100 \mathrm{mg} / \mathrm{dL}$ or 5.6 $\mathrm{mmol} / \mathrm{L}$, an oral glucose tolerance test is recommended, although it is not indispensable for diagnosing this syndrome. Central obesity is determined by measuring the waist circumference; its diagnosis depends on the person's sex and on the ethnic group to which the person belongs (not the person's country of residence), according to proposed cutoff points. The IDF consensus group also established additional parameters that should be used in research studies and recommended treatments for preventing the complications of diabetes and cardiovascular disease.

Key words: metabolic syndrome $X$, guidelines.

\section{REFERENCIAS}

1. Dunstan DW, Zimmet PZ, Welborn TA, De Courten MP, Cameron AJ, Sicree RA, et al. The rising prevalence of diabetes and impaired glucose tolerance. The Australian Diabetes, Obesity and Lifestyle Study. Diabetes Care. 2002;25: 829-34.

2. Isomaa $B$, Almgren $P$, Tuomi $T$, Forsen B, Lahti K, Nissen M, et al. Cardiovascular morbidity and mortality associated with the metabolic syndrome. Diabetes Care. 2001;24(4):683-9.

3. International Diabetes Federation. Diabetes atlas. 2. ${ }^{\mathrm{a}}$ ed. Brussels: IDF; 2003.

4. Expert Panel on Detection, Evaluation, and Treatment of High Blood Cholesterol in Adults. Executive summary of the third report of the National Cholesterol Education Program (NCEP) Expert Panel on Detection, Evaluation, and Treatment of High Blood Cholesterol in Adults (Adult Treatment Panel III). J Am Med Assoc. 2001;285(19):2486-97. Hallado en http://www.nhlbi.nih.gov/ guidelines/cholesterol/. Acceso el 21 de septiembre de 2005

5. Tan CE, Ma S, Wai D, Chew SK, Tai ES. Can we apply the National Cholesterol Education Program Adult Treatment Panel definition of the metabolic syndrome to Asians? Diabetes Care. 2004; 27:1182-6.

6. Chobanian AV, Bakris GL, Black HR, Cushman WC, Green LA, Izzo Jr JL, et al. Seventh report of the Joint National Committee on prevention, detection, evaluation, and treatment of high blood pressure. Hypertension. 2003; 42(6):1206-52

7. Knowler WC, Barrett-Connor E, Fowler SE, Hamman RF, Lachin JM, Walker EA, et al. Reduction in the incidence of type 2 diabetes with lifestyle intervention or metformin. N Engl J Med. 2002;346(6): 393-403.

8. Buchanan TA, Xiang AH, Peters RK, Kjos SL, Marroquin A, Goico J, et al. Preservation of pancreatic beta-cell function and prevention of type 2 diabetes by pharmacological treatment of insulin resistance in high-risk Hispanic women. Diabetes. 2002;51:2796-803.

9. Durbin RJ. Thiazolidinedione therapy in the prevention/delay of type 2 diabetes in patients with impaired glucose tolerance and insulin resistance. Diabetes Obes Metab. 2004;6:280-5.

10. Chiasson JL, Josse RG, Gomis R, Hanefeld M, Karasik A, Laakso M, et al. Acarbose treatment and the risk of cardiovascular disease and hypertension in patients with impaired glucose tolerance: the STOP-NIDDM trial. J Am Med Assoc. 2003;290(4):486-94.

11. Torgerson JS, Hauptman J, Boldrin MN, Sjöström L. XENical in the Prevention of Diabetes in Obese Subjects (XENDOS) Study. A randomized study of orlistat as an adjunct to lifestyle changes for the prevention of type 2 diabetes in obese patients. Diabetes Care. 2004;27: $155-61$. 